\title{
MONOTONE LINDELÖF PROPERTY AND LINEARLY ORDERED EXTENSIONS
}

\author{
AI-JUN XU ${ }^{凶}$ and WEI-XUE SHI
}

(Received 1 June 2009)

\begin{abstract}
In this paper, we explore the monotone Lindelöf property of two kinds of linearly ordered extensions of monotonically Lindelöf generalized ordered spaces. In addition, we construct nonseparable monotonically Lindelöf spaces using the Bernstein set, which generalizes Corollary 4 of Levy and Matveev ['Some more examples of monotonically Lindelöf and not monotonically Lindelöf spaces', Topology Appl. 154 (2007), 2333-2343].
\end{abstract}

2000 Mathematics subject classification: primary 54F05; secondary 54D20, 54D65.

Keywords and phrases: monotone Lindelöf property, linearly ordered extension, lexicographic product.

\section{Introduction}

Recently, Matveev introduced the concept of the monotone Lindelöf property. In [7] and [4], Levy and Matveev investigated the monotone Lindelöf property in countable spaces, and gave an example which is monotonically Lindelöf, but not monotonically normal. In [1], Bennett et al. presented basic results about the monotone Lindelöf property in the theory of generalized ordered spaces.

First, in this paper we show that the linearly ordered extension $X^{*}$ of a generalized ordered (GO)-space $X$ is monotonically Lindelöf if and only if $X$ is monotonically Lindelöf. However, it is not true for another linearly ordered extension $L(X)$ of a monotonically Lindelöf GO-space $X$ (see Example 2.6). In addition, we prove that if a GO-space $X$ is monotonically Lindelöf and $R \cup L$ is a countable subset of $X$, then $L(X)$ is monotonically Lindelöf, where $R=\{x \in X \mid[x, \rightarrow) \in \tau-\lambda\}$ and $L=\{x \in X \mid(\leftarrow, x] \in \tau-\lambda\}, \tau$ is a generalized ordered topology on $X$ and $\lambda$ is the interval topology on $X$. Second, we construct monotonically Lindelöf spaces using the Bernstein set and apply them to generalize the result in [3, Corollary 4].

We reserve the symbols $\mathbb{R}, \mathbb{Q}$ and $\mathbb{Z}$ for the sets of real numbers, rational numbers and integers. For a set $V$ and a collection $\mathcal{U}$ of sets, we write $V \prec \mathcal{U}$ to mean that $V$ is a subset of some member of $\mathcal{U}$.

This work is supported by the NSFC, project 10971092.

(C) 2010 Australian Mathematical Publishing Association Inc. 0004-9727/2010 \$16.00 
Recall that $X$ is monotonically Lindelöf if there is an operator $r$ assigning to every open cover $\mathcal{U}$ a countable open refinement $r \mathcal{U}$ (still covering the space) in such a way that $r \mathcal{V}$ refines $r \mathcal{U}$ whenever $\mathcal{V}$ refines $\mathcal{U}[3,7]$. In this case, $r$ will be called a monotone Lindelöf operator for the space $X$. A linearly ordered topological space (LOTS) is a triple $(X, \lambda, \leq)$, where $(X, \leq)$ is a linearly ordered set and $\lambda$ is the interval topology on $(X, \leq)$. A GO-space is a triple $(X, \tau, \leq)$, where $(X, \leq)$ is a linearly ordered set and $\tau$ is a topology on $(X, \leq)$ such that $\lambda \subseteq \tau$ and $\tau$ has a base consisting of order convex sets, where a set $A$ is called order convex if $x \in A$ for every $x$ lying between two points of $A$. If a GO-space $(X, \tau, \leq)$ can be topologically embedded in a LOTS $(Y, \lambda, \lessdot)$, then the LOTS $(Y, \lambda, \lessdot)$ is called an orderable extension of the GO-space $(X, \tau, \leq)$; if $\leq=\left.\lessdot\right|_{X}$, then the LOTS $(Y, \lambda, \lessdot)$ is called a linearly ordered extension of the GO-space $(X, \tau, \leq)$. In addition, if $(X, \tau, \leq)$ is dense (closed) in the space $(Y, \lambda, \lessdot)$, then $(Y, \lambda, \lessdot)$ is called a dense (closed) linearly ordered extension of the GO-space $(X, \tau, \leq)$. Let $X=(X, \tau, \leq)$ be a GO-space and $\lambda=\lambda(\leq)$ be the usual order topology on $X$. We set $R=\{x \in X \mid[x, \rightarrow) \in \tau-\lambda\}$ and $L=\{x \in X \mid(\leftarrow, x] \in \tau-\lambda\}$. Then $X^{*}$ is defined as follows:

$$
\begin{aligned}
X^{*}=( & X \times\{0\}) \cup\{\langle x, n\rangle \mid x \in R, n<0 \text { and } n \in \mathbb{Z}\} \\
& \cup\{\langle x, m\rangle \mid x \in L, m>0 \text { and } m \in \mathbb{Z}\} \subseteq X \times \mathbb{Z} .
\end{aligned}
$$

$L(X)$ is defined as follows:

$$
L(X)=(X \times\{0\}) \cup\{\langle x,-1\rangle \mid x \in R\} \cup\{\langle x, 1\rangle \mid x \in L\} \subseteq X \times\{-1,0,1\} .
$$

Then $X^{*}$ and $L(X)$ are LOTS equipped with the lexicographic order topologies (see [5, 8]). Observe that $X \times\{0\}$ as a subspace of $X^{*}$ and $L(X)$ is homeomorphic to $X$, so that we may identify $X \times\{0\}$ with $X$ and regard $X$ as a subspace of $X^{*}$ and $L(X)$ in this paper without further explanation. Therefore, it follows from the definitions of $X^{*}$ and $L(X)$ that $X$ is closed in $X^{*}$ and dense in $L(X)$, so $X^{*}$ is a closed linearly ordered extension of $X$ and $L(X)$ is a dense linearly ordered extension of $X$.

For undefined terminology and notation refer to $[2,5,6]$.

\section{Main results}

In this section we investigate the relationship between linearly ordered extensions and the monotone Lindelöf property. First, we have the following proposition that allows us to use open convex sets instead of open sets in the definition of monotonically Lindelöf spaces.

Proposition 2.1. For a GO-space $X$, the following conditions are equivalent.

(1) $X$ is monotonically Lindelöf.

(2) For any cover $\mathcal{U}$ of $X$ consisting of open convex subsets, there exists a countable open cover $r \mathcal{U}$ refining $\mathcal{U}$ such that if $\mathcal{V}$ is also such an open convex cover of $X$ that refines $\mathcal{U}$, then $r \mathcal{V}$ refines $r \mathcal{U}$. 
(3) For any cover $\mathcal{U}$ of $X$ consisting of open convex subsets, there exists a countable open cover $\mathrm{U} \mathcal{U}$ which also consists of convex subsets refining $\mathcal{U}$ such that if $\mathcal{V}$ is also such an open convex cover of $X$ that refines $\mathcal{U}$, then $r \mathcal{V}$ refines $r \mathcal{U}$.

Proof. That (1) implies (2) is obvious. To prove that (2) implies (3), let $r \mathcal{U}$ be the refinement of $\mathcal{U}$ satisfying the conditions in (2). Then members of $r \mathcal{U}$ may not be convex. For each member $O$ of $r \mathcal{U}$, define $C_{O}=\left\{x \in X \mid \exists x_{1}, x_{2} \in O\right.$ such that $x_{1} \leq$ $x \leq x_{2}$. Thus $C_{O}$ is convex and open such that if $O^{\prime} \subset O$, then $C_{O^{\prime}} \subset C_{O}$. Moreover, if $U$ is an open convex subset that contains $O$, then $C_{O} \subset U$. Put $r^{\prime} \mathcal{U}=\left\{C_{O} \mid\right.$ $O \in r \mathcal{U}$ \}. Then $r^{\prime} \mathcal{U}$ satisfies the conditions in (3). Now we prove that (3) implies (1). Suppose that $\mathcal{U}$ is an open cover of $X$ whose members may not be convex. For each member $U$ of $\mathcal{U}$, let $\mathcal{O}(U)=\{O \mid O$ is a convex component of $U\}$. Then $\mathcal{U}^{\prime}=\cup\{\mathcal{O}(U) \mid U \in \mathcal{U}\}$ is an open cover of $X$ consisting of convex subsets and it refines $\mathcal{U}$ so that if $\mathcal{V}$ refines $\mathcal{U}$, then $\mathcal{V}^{\prime}$ refines $\mathcal{U}^{\prime}$. By (3), $r \mathcal{V}^{\prime}$ refines $r \mathcal{U}^{\prime}$. Therefore, $X$ is monotonically Lindelöf.

LeMma 2.2 [5]. Suppose that $\mathcal{C}$ is an open cover of a $G O$-space $X$ by convex sets. Let $E=\{x \in X \mid$ no element of $\mathcal{C}$ contains points on both sides of $x\}$. Then for each $y \in X$ there is an open neighbourhood $G(y)$ of $y$ such that $G(y) \cap E \subseteq\{y\}$.

Let $S \subseteq X$ be convex in a GO-space $X$. In [5], Lutzer defined

$$
S^{\sim}=\left\{\langle x, k\rangle \in X^{*} \mid x \in I(S)\right\} \cup\{\langle x, 0\rangle \mid x \in S-I(S)\}
$$

where

$$
k \in \mathbb{Z} \quad \text { and } \quad I(S)=\{x \in S \mid \exists a, b \in S \text { with } a<x<b\} .
$$

Using $L(X)$ in place of $X^{*}$, we define

$$
S^{-}=\{\langle x, k\rangle \in L(X) \mid x \in I(S)\} \cup\{\langle x, 0\rangle \mid x \in S-I(S)\}
$$

where $k=-1,0$ or 1 and $I(S)$ is defined as before.

It is well known that any nonvoid (open) subset $G$ of a GO-space $X$ can be uniquely represented as a union of its maximal (open) convex subsets, which are called convex components of $G$.

REMARK 2.3. Let $G$ be a subset of a GO-space $X$. If $G=\emptyset$, let $G^{\sim}=\emptyset\left(G^{-}=\emptyset\right)$. If $G \neq \emptyset$, let $G=\cup\left\{S_{i} \mid i \in I\right\}$ be the unique representation of $G$ as a union of its convex components. Put $G^{\sim}=\cup\left\{S_{i}^{\sim} \mid i \in I\right\}\left(G^{-}=\cup\left\{S_{i}^{-} \mid i \in I\right\}\right)$.

Proposition 2.4 [5]. Let $X$ be a $G O$-space.

(1) If $G \subseteq H \subseteq X$, then $G^{\sim} \subseteq H^{\sim}$.

(2) If $G$ is open in $X$, then $G^{\sim}$ is open in $X^{*}$.

(3) If $\mathcal{C}$ is a collection of convex subsets of $X^{*}$ and if $\mathcal{G}$ is a collection of subsets of $X$ which refines $\mathcal{C}$, then so does the collection $\mathcal{G}^{\sim}=\left\{G^{\sim} \mid G \in \mathcal{G}\right\}$.

By definition, the LOTS $X^{*}(L(X))$ is a closed (dense) linearly ordered extension of a GO-space $X$. It is interesting to investigate the following question. 
$(\dagger$ ) Does the linearly ordered extension of a GO-space $X$ have a property $\mathrm{P}$ when $X$ has the property $\mathrm{P}$ ?

For the monotone Lindelöf property, we shall prove that a GO-space $X$ is monotonically Lindelöf if and only if $X^{*}$ is monotonically Lindelöf.

THEOREM 2.5. Let $X$ be a GO-space. Then the following are equivalent:

(1) $X$ is a monotonically Lindelöf space;

(2) $X^{*}$ is a monotonically Lindelöf space.

Proof. (1) implies (2). Let $\mathcal{C}$ be an open cover of $X^{*}$. By Proposition 2.1, we may assume that every member of $\mathcal{C}$ is convex. Then $\mathcal{U}_{\mathcal{C}}=\{C \cap X \mid C \in \mathcal{C}\}$ is an open cover of $X$ by convex sets, so by Proposition 2.1 there is a countable open cover $r_{1} \mathcal{U}_{\mathcal{C}}$ of $X$ that refines $\mathcal{U}_{\mathcal{C}}$, where $r_{1}$ is a monotone Lindelöf operator for the space $X$ and every member of $r_{1} \mathcal{U}_{\mathcal{C}}$ is convex. Define

$$
E_{\mathcal{C}}=\left\{x \in X \mid \text { no element of } r_{1} \mathcal{U}_{\mathcal{C}} \text { contains points on both sides of } x\right\} .
$$

By Lemma 2.2, $E_{\mathcal{C}}$ is a closed discrete subspace of $X$. Since $X$ is monotonically Lindelöf, $E_{\mathcal{C}}$ must be countable. Let $\mathcal{H}_{\mathcal{C}}=\left\{V^{\sim} \mid V \in r_{1} \mathcal{U}_{\mathcal{C}}\right\}$. By Proposition 2.4(2) and $(3), \mathcal{H}_{\mathcal{C}}$ is a countable open collection of $X^{*}$, and $\mathcal{H}_{\mathcal{C}}$ refines $\mathcal{U}_{\mathcal{C}}$ and $\mathcal{C}$ since $r_{1} \mathcal{U}_{\mathcal{C}}$ refines $\mathcal{U}_{\mathcal{C}}$. In addition, if $\langle x, k\rangle \in X^{*}-\cup \mathcal{H}_{\mathcal{C}}$, then $x \in E_{\mathcal{C}}$, where $x \in X$ and $k \in \mathbb{Z}-\{0\}$. Hence, $X^{*}-\cup \mathcal{H}_{\mathcal{C}}$ is a countable set. Let $r_{2} \mathcal{C}=\{\{\langle x, k\rangle\} \mid\langle x, k\rangle \in$ $\left.X^{*}-\cup \mathcal{H}_{\mathcal{C}}\right\}$. Therefore, $\mathcal{C}$ has a countable refinement $r \mathcal{C}=\mathcal{H}_{\mathcal{C}} \cup r_{2} \mathcal{C}$.

Finally, suppose that $\mathcal{C}$ and $\mathcal{D}$ are open covers of $X^{*}$ and $\mathcal{C}$ refines $\mathcal{D}$. Similar to before, for $\mathcal{D}$, we may define $\mathcal{H}_{\mathcal{D}}, \mathcal{U}_{\mathcal{D}}, r_{1} \mathcal{U}_{\mathcal{D}}, E_{\mathcal{D}}, r_{2} \mathcal{D}, r \mathcal{D}$ corresponding to $\mathcal{H}_{\mathcal{C}}, \mathcal{U}_{\mathcal{C}}, r_{1} \mathcal{U}_{\mathcal{C}}, E_{\mathcal{C}}, r_{2} \mathcal{C}, r \mathcal{C}$. Hence, $\mathcal{G}_{\mathcal{C}}$ refines $\mathcal{G}_{\mathcal{D}}$. Then $\mathcal{U}_{\mathcal{C}}$ refines $\mathcal{U}_{\mathcal{D}}$ and $r_{1} \mathcal{U}_{\mathcal{C}}$ refines $r_{1} \mathcal{U}_{\mathcal{D}}$. By Proposition 2.4(1), $\mathcal{H}_{\mathcal{C}}$ refines $\mathcal{H}_{\mathcal{D}}$. Then $E_{\mathcal{C}} \supseteq E_{\mathcal{D}}$. So any member of $r_{2} \mathcal{C}$ is either a member of $r_{2} \mathcal{D}$ or a subset of some member of $\mathcal{H}_{\mathcal{D}}$. Hence, $r \mathcal{C}$ refines $r \mathcal{D}$. Therefore, $X^{*}$ is monotonically Lindelöf.

That (2) implies (1) follows directly from $X$ being a closed subset of $X^{*}$.

From the following example we know that, for some monotonically Lindelöf GOspaces $X, L(X)$ may not be monotonically Lindelöf.

EXAmple 2.6. There exists a GO-space $X$ that is monotonically Lindelöf, but $L(X)$ is not so.

PROOF. Let $X$ be $\omega_{1}+1$ with a topology such that every point of $\omega_{1}$ is isolated, and every neighbourhood of $\omega_{1}$ is a usual neighbourhood.

(1) $X$ is monotonically Lindelöf. For any open cover $\mathcal{U}$ of $X$, there is a first ordinal $\alpha=\alpha(\mathcal{U})$ such that the interval $\left[\alpha, \omega_{1}\right]$ is a subset of some member of $\mathcal{U}$. Define $r \mathcal{U}=\left\{\left[\alpha, \omega_{1}\right]\right\} \cup\{\{\beta\} \mid \beta<\alpha\}$. Clearly, $r$ is a monotone Lindelöf operator for the space $X$.

(2) $L(X)$ is not monotonically Lindelöf. In fact, $L(X)$ is homeomorphic to $\omega_{1}+1$ with the usual interval topology since $L(X)$ may be regarded as being made from $\omega_{1}+1$ by inserting a predecessor at each limit ordinal less than $\omega_{1}$, but these inserted 
'predecessors' play the role of the limit ordinals instead. By [1, Example 2.3], the usual ordinal space $\omega_{1}+1$ is not monotonically Lindelöf. Thus $L(X)$ is not monotonically Lindelöf.

Example 2.6 motivates the following question.

( ) Under what conditions on a monotonically Lindelöf GO-space $X$ must $L(X)$ be monotonically Lindelöf?

We give an answer in Theorem 2.8 below.

Proposition 2.7. Let $X$ be a $G O$-space.

(1) If $S \subseteq T$ is convex in $X$, then $S^{-} \subseteq T^{-}$.

(2) If $S$ is convex in $X$, then $S^{-}$is open in $L(X)$ if and only if $S$ is open in $X$.

(3) If $H$ is convex in $L(X)$ and if $S \subseteq H$, where $S$ is convex in $X$, then $S^{-} \subseteq H$.

(4) If $G$ is open in $X$, then $G^{-}$is open in $L(X)$.

(5) If $\mathcal{D}$ is a collection of convex subsets of $L(X)$ and if $\mathcal{C}$ is a collection of subsets of $X$ which refines $\mathcal{D}$, then so does the collection $\mathcal{C}^{-}=\left\{C^{-} \mid C \in \mathcal{C}\right\}$.

PROOF. We only prove (2). The other results can be shown similarly to the proofs of [5, Lemma 3.2 and Proposition 3.5]. Suppose that $S$ is an open convex set in $X$. Let $\langle x, k\rangle \in S^{-}$, where $k=0,1,-1$. If $x \in I(S)$, choose points $a, b \in S$ with $a<$ $x<b$. Then $\langle x, k\rangle \in(\langle a, 0\rangle,\langle b, 0\rangle) \subseteq S^{-}$. If $x \notin I(S)$, then either $x \in S \subseteq[x, \rightarrow)$ or $x \in S \subseteq(\leftarrow, x]$. In either case, $k=0$. For the first case, there are two subcases:

(i) $\quad x$ is an isolated point of $X$. Then $\{\langle x, 0\rangle\}$ is an open subset of $L(X)$ which is contained in $S^{-}$.

(ii) $\quad x$ is not an isolated point of $X$. Since $S$ is open in $X$, either $x$ has an immediate predecessor $y \in X$ (in which case we let $p=\langle y, 0\rangle$ ), or else the point $p=\langle x,-1\rangle \in L(X)$. By assumption, there are two points $x_{1}$ and $x_{2} \in S$ with $x<x_{1}<x_{2}$. Let $q=\left\langle x_{1}, 0\right\rangle$. Then $\langle x, 0\rangle \in(p, q) \subseteq S^{-}$and hence $S^{-}$is open in $L(X)$.

The converse is clear because $S^{-} \cap X=S$.

THEOREM 2.8. If $X$ is a monotonically Lindelöf $G O$-space and $R \cup L$ is countable, then $L(X)$ is monotonically Lindelöf.

Proof. Suppose that $\mathcal{C}$ is an open cover of $L(X)$. As in the proof of Theorem 2.5, we have $\mathcal{G}_{\mathcal{C}}, \mathcal{U}_{\mathcal{C}}, r_{1} \mathcal{U}_{\mathcal{C}}$, where

$$
\mathcal{G}_{\mathcal{C}}=\{G \mid G \text { is a convex component of some member } C \text { of } \mathcal{C}\}
$$

and

$$
\mathcal{U}_{\mathcal{C}}=\left\{G \cap X \mid G \in \mathcal{G}_{\mathcal{C}}\right\}
$$

and $r_{1} \mathcal{U}_{\mathcal{C}}$ refines $\mathcal{U}_{\mathcal{C}}$, where $r_{1}$ is a monotone Lindelöf operator for the space $X$.

Let $\mathcal{H}_{\mathcal{C}}=\left\{V^{-} \mid V \in r_{1} \mathcal{U}_{\mathcal{C}}\right\}$. In the light of Proposition 2.7(2) and (5) and $r_{1} \mathcal{U}_{\mathcal{C}}$ refining $\mathcal{G}_{\mathcal{C}}, \mathcal{H}_{\mathcal{C}}$ is a countable open collection of $L(X)$ that refines $\mathcal{G}_{\mathcal{C}}$. Since $\mathcal{G}_{\mathcal{C}}$ refines $\mathcal{C}, \mathcal{H}_{\mathcal{C}}$ refines $\mathcal{C}$. If $\langle x, k\rangle \in L(X)-\cup \mathcal{H}_{\mathcal{C}}$, then $k$ must be -1 or 1 . So either $x \in R$, if $k=-1$, or $x \in L$, if $k=1$. By the definition of $R$ (respectively $L$ ), 
$x$ does not have an immediate predecessor (respectively, successor) in $X$, for every $x \in R$ (respectively $x \in L$ ). Hence, $\langle x,-1\rangle$ (respectively $\langle x, 1\rangle$ ) does not have an immediate predecessor (respectively, successor) in $L(X)$, for every $x \in R$ (respectively $x \in L)$. Suppose that $W$ is any well-order of the underlying set $X$. For every $x \in R$, let $Y_{\mathcal{C} 1}(x)=\left\{y \in X \mid\langle y, 0\rangle \in \operatorname{st}\left(\langle x,-1\rangle, \mathcal{G}_{\mathcal{C}}\right), y<x\right\}$, where $\operatorname{st}\left(\langle x,-1\rangle, \mathcal{G}_{\mathcal{C}}\right)=$ $\cup\left\{G \in \mathcal{G}_{\mathcal{C}} \mid\langle x,-1\rangle \in G\right\}$. Let $x_{\mathcal{C} 1} \in X$ be the $W$-first point of the set $Y_{\mathcal{C} 1}(x)$. Then there exists $G \in \mathcal{G}_{\mathcal{C}}$ such that $\left(\left\langle x_{\mathcal{C} 1}, 0\right\rangle,\langle x,-1\rangle\right] \subseteq G$. Similarly, for every $x \in L$, we define $Y_{\mathcal{C} 2}(x), x_{\mathcal{C} 2}$ corresponding to $Y_{\mathcal{C} 1}(x), x_{\mathcal{C} 1}$. Then there exists $G \in \mathcal{G}_{\mathcal{C}}$ such that $\left[\langle x, 1\rangle,\left\langle x_{\mathcal{C} 2}, 0\right\rangle\right) \subseteq G$. Define $r \mathcal{C}=\mathcal{H}_{\mathcal{C}} \cup\left\{\left(\left\langle x_{\mathcal{C} 1}, 0\right\rangle,\langle x,-1\rangle\right] \mid x \in R\right\} \cup$ $\left\{\left[\langle x, 1\rangle,\left\langle x_{\mathcal{C} 2}, 0\right\rangle\right) \mid x \in L\right\}$. Then $r \mathcal{C}$ is a countable open cover of $L(X)$, which refines $\mathcal{G}_{\mathcal{C}}$ and $\mathcal{C}$.

Suppose that $\mathcal{C}$ and $\mathcal{D}$ are open covers of $L(X)$ and that $\mathcal{C}$ refines $\mathcal{D}$. Clearly, $\mathcal{H}_{\mathcal{C}}$ refines $\mathcal{H}_{\mathcal{D}}$. Since $\mathcal{G}_{\mathcal{C}}$ refines $\mathcal{G}_{\mathcal{D}}, Y_{\mathcal{C} 1}(x) \subseteq Y_{\mathcal{D} 1}(x)$.

We claim that $x_{\mathcal{D} 1} \leq x_{\mathcal{C} 1}$ for every $x \in R$. In fact, if $x_{\mathcal{D} 1}>x_{\mathcal{C} 1}$, then $x_{\mathcal{D} 1} \in Y_{\mathcal{C} 1}(x)$. Hence, $x_{\mathcal{D} 1}$ follows $x_{\mathcal{C} 1}$ under the well-order $W$. However, $x_{\mathcal{C} 1}$ follows $x_{\mathcal{D} 1}$ under the well-order $W$ since $x_{\mathcal{C} 1}, x_{\mathcal{D} 1} \in Y_{\mathcal{D} 1}(x)$. This is a contradiction.

By the above claim, $\left(\left\langle x_{\mathcal{C} 1}, 0\right\rangle,\langle x,-1\rangle\right] \subseteq\left(\left\langle x_{\mathcal{D} 1}, 0\right\rangle,\langle x,-1\rangle\right]$. Similarly, for every $x \in L$, we have $\left[\langle x, 1\rangle,\left\langle x_{\mathcal{C} 2}, 0\right\rangle\right) \subseteq\left[\langle x, 1\rangle,\left\langle x_{\mathcal{D} 2}, 0\right\rangle\right)$. Therefore, $r \mathcal{C}$ refines $r \mathcal{D}$.

In Theorem 2.8, the condition ' $R \cup L$ is countable' is not necessary. For example, let $S$ be the Sorgenfrey line. Then $S$ and $L(S)$ are monotonically Lindelöf since they are separable, but the subset $R \subseteq S$ is uncountable.

Finally, we generalize the result of [3, Corollary 4] in Theorem 2.9. By [1, Proposition 3.1], the Sorgenfrey line is monotonically Lindelöf and the Michael line is not. In [3], Levy and Matveev showed that $\left(\mathbb{R}, \mathcal{E}_{B}\right)$ is monotonically Lindelöf, where $B$ is a Bernstein subset of the real line $\mathbb{R}$ with the usual topology $\mathcal{E}$, and $\mathcal{E}_{B}=$ $\mathcal{E} \cup\{\{b\} \mid b \in B\}$. A subset $A$ of $\mathbb{R}$ is called a Bernstein set if $|A|=\mathfrak{c}$ (continuum) and every compact set contained either in $A$ or in $\mathbb{R}-A$ is countable.

THEOREM 2.9. Let $A$ be a Bernstein set of the real line $\mathbb{R}$ and let $\lambda$ be the usual topology on $\mathbb{R}$. If $\rho$ is a generalized ordered topology on $\mathbb{R}$ that is stronger than the usual topology $\lambda$ and has a neighbourhood base at each point of A consisting of usual open intervals, then $Y=(\mathbb{R}, \rho)$ is monotonically Lindelöf.

PROOF. Let $\mathcal{U}$ be an open cover of $Y$. Define $r_{1} \mathcal{U}=\{(a, b) \mid(a, b) \prec \mathcal{U}$ and $a, b \in$ $\mathbb{Q}\}$. Then $r_{1} \mathcal{U}$ is a countable open collection and partially refines $\mathcal{U}$. In addition, $r_{1} \mathcal{U}$ covers $A$ and $\cup r_{1} \mathcal{U}$ is an open subset of $\mathbb{R}$ with respect to the usual interval topology. Then the set $\mathbb{R}-\cup r_{1} \mathcal{U}$ is disjoint from $A$ and a closed set of $\mathbb{R}$ with respect to the usual interval topology. By the definition of a Bernstein set, $\mathbb{R}-\cup r_{1} \mathcal{U}$ is countable. Define

$$
\begin{gathered}
r_{2} \mathcal{U}=\left\{\{y\} \mid y \in \mathbb{R}-\cup r_{1} \mathcal{U},\{y\} \in \rho\right\}, \\
Y_{1}(\mathcal{U})=\{y \in \mathbb{R} \mid y \text { is not an isolated point and }(\leftarrow, y] \in \rho\},
\end{gathered}
$$

and

$$
Y_{2}(\mathcal{U})=\{y \in \mathbb{R} \mid y \text { is not an isolated point and }[y, \rightarrow) \in \rho\}
$$


For every $y \in Y_{1}(\mathcal{U})$, let

$$
F_{1}(y, \mathcal{U})=\{q \in \mathbb{Q} \mid q<y \text { and }(q, y] \prec \mathcal{U}\},
$$

and

$$
G_{1}(y, \mathcal{U})=\{q \in \mathbb{Q} \mid \exists z>y \text { and }(q, z) \prec \mathcal{U}\} .
$$

Then $G_{1}(y, \mathcal{U}) \subseteq F_{1}(y, \mathcal{U})$. Let $D_{1}(y, \mathcal{U})=F_{1}(y, \mathcal{U})-G_{1}(y, \mathcal{U})$. Define

$$
r_{3} \mathcal{U}=\left\{(q, y] \mid y \in Y_{1}(\mathcal{U}), D_{1}(y, \mathcal{U}) \neq \emptyset \text { and } q \in F_{1}(y, \mathcal{U})\right\} .
$$

For every $y \in Y_{2}(\mathcal{U})$, analogously define $F_{2}(y, \mathcal{U}), G_{2}(y, \mathcal{U})$ and $D_{2}(y, \mathcal{U})$ and $r_{4} \mathcal{U}=$ $\left\{[y, q) \mid y \in Y_{2}(\mathcal{U}), D_{2}(y, \mathcal{U}) \neq \emptyset\right.$ and $\left.q \in F_{2}(y, \mathcal{U})\right\}$. Let $r \mathcal{U}=\cup\left\{r_{i} \mathcal{U} \mid 1 \leq i \leq 4\right\}$. The remainder of the proof follows [1, Proposition 3.1].

By Theorem 2.9 we have the same conclusion as [3, Corollary 4].

Corollary 2.10. Suppose that $Y=\mathbb{R}$ is topologized by isolating the points of $\mathbb{R}-A$ and leaving the points of $A$ with their usual neighbourhoods. Then $Y$ is monotonically Lindelöf.

\title{
Acknowledgement
}

The authors would like to express their thanks to the referee for valuable suggestions that improved this paper.

\section{References}

[1] H. Bennett, D. Lutzer and M. Matveev, 'The monotone Lindelöf property and separability in ordered spaces', Topology Appl. 151 (2005), 180-186.

[2] R. Engleking, General Topology (Sigma Series in Pure Mathematics, 6) (Heldermann, Berlin, 1989), revised.

[3] R. Levy and M. Matveev, 'Some more examples of monotonically Lindelöf and not monotonically Lindelöf spaces', Topology Appl. 154 (2007), 2333-2343.

[4] R. Levy and M. Matveev, 'The monotone Lindelöfness of countable spaces', Comment. Math. Univ. Carolin. 49(1) (2008), 155-161.

[5] D. Lutzer, 'On generalized ordered spaces', Dissertationes Math. Rozprawy Mat. 89 (1971), 32 pp.

[6] D. Lutzer, 'Ordered topological spaces', in: Surveys in General Topology, (ed. G. M. Reed) (Academic Press, New York, 1980), pp. 247-296.

[7] M. Matveev, A monotonically Lindelöf space which is not monotonically normal, Preprint.

[8] T. Miwa and N. Kemoto, 'Linearly ordered extensions of GO-spaces', Topology Appl. 54 (1993), $133-140$.

\author{
AI-JUN XU, Department of Applied Mathematics, Nanjing Forest University, \\ Nanjing 210037, PR China \\ e-mail: ajxu@njfu.edu.cn
}

WEI-XUE SHI, Department of Mathematics, Nanjing University, Nanjing 210093, PR China

e-mail:wxshi@nju.edu.cn 